\title{
Local interstellar cloud grains outside the heliopause
}

\author{
A. Czechowski ${ }^{1}$ and I. Mann ${ }^{2}$ \\ 1 Space Research Centre, Polish Academy of Sciences, Bartycka 18A, 00-716 Warsaw, Poland \\ 2 Institut fuer Planetologie, Westfaelische Wilhelms-Universitaet, Wilhelm-Klemm-Str. 10, 48149 Muenster, Germany
}

Received 31 January 2003 / Accepted 18 July 2003

\begin{abstract}
The dynamics of small $(\sim 0.01 \mu \mathrm{m})$ interstellar grains in the heliospheric transition region is studied using numerical simulations. The velocity distributions of the grains at the boundary of the heliosphere are found to depend sensitively on the global structure of the transition region, in particular on the presence of the heliospheric bow shock, and can be also used to estimate the charge-to-mass ratio of grains. The simulations include the grain impact velocity distributions that could be measured on board an Interstellar Probe spacecraft. Similar dust dynamics is expected around astrospheres.
\end{abstract}

Key words. ISM: dust, extinction - ISM: kinematics and dynamics - magnetic fields - interplanetary medium Sun: solar wind

\section{Introduction}

The interaction of the solar wind with the surrounding interstellar medium is seen through its effect on solar system phenomena, like modulation of the fluxes of galactic and anomalous cosmic rays, production of energetic neutral atoms and the filtering of interstellar neutrals and interstellar dust that enter the solar system. But the structure of the transition region between the solar plasma environment (the heliosphere) and the interstellar medium is mostly not probed by direct observations. From theoretical considerations supported by model simulations (Baranov \& Malama 1993; Pauls et al. 1995; Pogorelov 1995; Washimi \& Tanaka 1996; Zank et al. 1996; Ratkiewicz et al. 1998, 2000; Linde et al. 1998; Pogorelov \& Matsuda 1998; Tanaka \& Washimi 1999; Fahr et al. 2000; see also von Steiger et al. 1996) there emerges a picture that is qualitatively consistent with the early model of Parker (1963): the solar wind passes through a shock transition (the termination shock) at which it decelarates to subsonic speed. Outside of the shock the flow has a stagnation point structure with the plasma of solar origin separated from the interstellar plasma by a discontinuity surface, the heliopause. The simulations (those that include the plasma-neutral component interaction) give the Sun-termination shock distance of the order of $100 \mathrm{AU}$ in the apex direction, and the distance from the Sun to the nose of the heliopause of the order of $150 \mathrm{AU}$. The models suggest that the heliospheric bow shock should exist in the interstellar flow in front of the heliosphere, although this conclusion depends on assumptions about the parameters of the interstellar medium (Zank et al. 1996). A region of enhanced temperature and density (the hydrogen wall), caused by charge exchange

Send offprint requests to: A. Czechowski, e-mail: ace@cbk.waw.pl with plasma in the transition region, is predicted in front of the heliosphere (Baranov \& Malama 1993).

Knowledge of some of the parameters characterizing the local interstellar medium has improved during the past decade. The study of interstellar absorption lines that are superimposed on the spectra of nearby stars allows one to determine the column densities of some of the elements in the interstellar medium as well as the velocity of the Local Interstellar Cloud (LIC) (cf. Lallement et al. 1995) relative to the Sun. Astronomical observations also proved the existence of a hydrogen wall in front of the heliosphere (Linsky \& Wood 1996). The hydrogen walls were also observed around nearby stars (Wood \& Linsky 1998) leading to the discussion of so-called astrospheres as circumstellar analogues to the heliosphere (cf. Lallement 2001).

In situ measurements aboard the Voyager and Ulysses spacecrafts greatly improved our understanding of the heliosphere and the surrounding interstellar medium. The heliospheric radio emissions in the $2-3 \mathrm{kHz}$ range detected by Voyagers suggest that the electron density in the local interstellar medium (LISM) is $0.04 \mathrm{~cm}^{-3}$ (Gurnett et al. 1993), and, from the directional analysis of these data (Kurth et al. 2003), that the local interstellar magnetic field lies in the galactic plane. Ulysses results include the direct measurement of interstellar He entering the heliosphere which allowed investigators to determine the velocity vector of the interstellar wind (Witte et al. 1993). A further important result was the detection of interstellar dust aboard Ulysses. While there were some early indications for the existence of interstellar dust in the solar system (Bertaux \& Blamont 1976), the path of the Ulysses spacecraft (Wenzel et al. 1992), almost perpendicular to the interstellar wind flux entering the solar system, allowed one to clearly distinguish the interstellar dust from solar system 
components (Grün et al. 1994) and for almost a decade now the Ulysses dust experiment has provided a detailed data set (Grün et al. 2000; Krueger et al. 2001).

The interstellar dust detected in the heliosphere covers only the large end of the size distribution. There are several deflection mechanisms that act selectively within the solar system on dust particles of certain sizes and properties (Levy \& Jokipii 1976; Mann 1996; Mann \& Kimura 2000). Very small dust particles gain a high charge to mass ratio (Kimura \& Mann 1998) and are already deflected outside the heliosphere. Current model calculations (Linde \& Gombosi 2000) of the filtration of these small dust particles do not yet agree with the dust sizes measured in the solar system, the Ulysses data indicating that small grains are more strongly suppressed (Linde $\&$ Gombosi 2000). Knowledge of the size distribution and the composition of small dust in the interstellar medium (ISM), which would allow one to constrain further the astrophysical models of the dust evolution in the ISM, cannot be directly obtained from the observations in the solar system. Therefore in-situ analysis of the smaller grains is an important task for space missions to the boundaries of the solar system. An interstellar probe mission was suggested to NASA (cf. Mewaldt \& Liewer 2001) and is in long term planning. The study of interstellar dust would be one of the goals of the interstellar probe mission.

The main objective of the dust measurements planned for the Interstellar Probe is to study the composition of the grains. If the procedure involves measuring the impact speed of the grain, the distribution in impact speed can be derived without additional instrumentation. In addition, the distribution of impact directions can be approximately obtained by scanning different directions by spacecraft rotation.

The velocity distributions of the grains in the interstellar medium would be of fundamental interest. It is also important to understand how these distributions would be affected by the passage of the grains through the transition region between the interstellar medium and the heliosphere. In the following we show that small interstellar grains (including the grains in the $0.01 \mu \mathrm{m}$ range, which are probably over the detection threshold of the probe) can be used as a sensitive indicator of the global structure of the transition region, in particular of the presence of sharp shocks. Also, when taken together with the magnetic field and plasma measurements, the grain velocity distributions in the transition region provide the possibility of estimating the electric charge on small interstellar grains.

To study these effects, we have developed a model of the heliospheric transition region that is simple yet adequate given the uncertainties of the parameters that have to be considered. This model we use for simulating the dynamics of small interstellar grains. In particular, we estimate the fluxes and the impact velocity distributions of small grains that could be measured aboard the interstellar probe and their dependence on the structure of the transition region. Our model is also applicable to astrospheres, which will be the target of a future study. Some of our results, concerning the large-scale streaming of the dust around the heliopause, may be interesting from this point of view.

\section{Interstellar grains and the heliospheric transition region}

The structure of the transition region between the solar wind and the local interstellar medium is still not known from direct observation and can only be deduced from theoretical arguments, with some support from the data (cosmic ray modulation, heliospheric radio emissions, Lyman- $\alpha$ measurements). The main expected features are the solar wind termination shock, the heliopause separating the plasmas of solar and of interstellar origin, and (depending on the detailed values of the parameters of the Local Interstellar Medium (LISM)) the heliospheric bow shock. The unknowns include the direction and the magnitude of the local interstellar magnetic field, although some estimations have been done (Frisch 1996; see also the Introduction).

The change in the plasma flow pattern from the largely uniform flow in the LISM to the transition region must affect the velocity distribution of the grains. The effect will be small for large grains, with a Larmor rotation time $T_{\mathrm{L}}=2 \pi / \Omega_{\mathrm{L}}$ $\left(\Omega_{\mathrm{L}}=Q|\boldsymbol{B}| / m c\right)$ much larger than the characteristic time of the flow $T_{\mathrm{f}} \sim L / V\left(L \sim 10^{2} \mathrm{AU}, V \sim 10 \mathrm{~km} \mathrm{~s}^{-1}\right.$ giving $T_{\mathrm{f}} \sim 1.5 \times 10^{9} \mathrm{~s}$ ). However, the grains of size of the order of $0.01 \mu \mathrm{m}$ and mass $\sim 10^{7} \mathrm{~m}_{\mathrm{p}}$, if charged to $\sim 10$ units of e, will have $T_{\mathrm{L}}$ of the order of $3 \times 10^{8} \mathrm{~s}$ (taking $|\boldsymbol{B}| \sim 2 \mu \mathrm{G}$ in the transition region) and for still smaller grains $T_{\mathrm{L}} \ll T_{\mathrm{f}}$ will hold (for $0.001 \mu \mathrm{m}$ grains we estimate $T_{\mathrm{L}}$ to be of the order of $\left.10^{6} \mathrm{~s}\right)$. The switch from the form of the velocity distribution typical for small (large charge/mass ratio) grains would occur for the Larmor rotation time approximately equal to the flow time. By looking at the size dependence of the observed distributions, one may therefore be able to estimate the charge on the grains.

To see how the velocity distributions of the grains could be modified by passage through the transition region, we assume the simplest stationary-flow picture of the transition region, with turbulence and wave effects disregarded. Apart from possible sharp shocks (like bow shock) the variations of the plasma flow and the magnetic field over the transition region would, from the point-of-view of small $\left(T_{\mathrm{L}} \ll T_{\mathrm{f}}\right)$ grains, occur slowly enough to be essentially adiabatic: the grains are able to adjust to the change in the plasma motion. If the initial grain velocity relative to the plasma would be small, it would not (for $T_{\mathrm{L}} \ll T_{\mathrm{f}}$ grains) much increase during the passage through the "adiabatic" region.

On the other hand, small grains would acquire a velocity difference relative to the plasma, with the corresponding increase in rigidity and in Larmor rotation radius, when crossing shock waves (because of low velocity of the grains, it is necessary to use the grain velocity in the plasma frame when defining the rigidity and the Larmor radius). The bow shock, if sharp enough, would be of particular importance. It follows that the velocity distribution of small grains in the transition region is very sensitive to the presence of sharp shocks.

Below we discuss the passage of small grains through the transition region, using numerical simulations to derive the velocity distributions of the grains at the points corresponding to a path that aims toward the nose of the heliosphere, i.e. the 
interstellar upwind direction, similar to that considered for the Interstellar Probe. We do not consider here the problem of penetration of the interstellar grains into the solar system (Linde \& Gombosi 2000). As an additional application of our method, although its relation to possible observations is more distant, we consider the global structure of the distribution of small grains around the boundary of the heliosphere. The motion of small grains reflects the structure of the magnetic field at the heliospheric boundary. The model of the heliosphere used in our simulations is simplified, but we can derive some qualitative conclusions.

\section{The method}

\subsection{Equations}

The equation of motion for a charged dust particle is:

$\frac{\mathrm{d} \boldsymbol{v}}{\mathrm{d} t}=\frac{Q}{m c}\left(\boldsymbol{v}-\boldsymbol{v}_{p}\right) \times \boldsymbol{B}+\frac{\boldsymbol{F}}{m}$.

Here $\boldsymbol{v}, m$ and $Q$ are the velocity, the mass and the charge of the grain, $\boldsymbol{B}$ is the magnetic field (we disregard the possible small-scale inhomogeneities), $\boldsymbol{v}_{\mathrm{p}}$ the velocity of the plasma flow (which induces the electric field $-(1 / c) \boldsymbol{v}_{\mathrm{p}} \times \boldsymbol{B}$ in the fixed frame) and $\boldsymbol{F}$ denotes the remaining forces acting on a grain (gravity, radiation pressure, gas drag) which are negligible for small grains in the transition region.

The charge $Q$ of the grain varies in time. This can be described by the equation

$\frac{\mathrm{d} Q}{\mathrm{~d} t}=\sum_{i} J_{i}$.

Here $J_{i}=J_{i}(\boldsymbol{x}, Q)$ denotes the charging rates contributing to the net charge on the grain (since grain velocity relative to plasma is small, we omit the dependence of the charging rates on the grain velocity). For small grains, with the average charge of the order of a single unit of e, the quantization of charge should be taken into account: Eq. (2) should in that case be considered as a stochastic equation. However, if the jumps in charge occur with frequency significantly higher than the Larmor frequency, one can take $Q$ to be time-averaged over a period of time large compared to charge-fluctuation time and treat Eq. (2) approximately as a differential equation. In the LISM this would likely apply to the grains of the size of $0.01 \mu \mathrm{m}$ (charge fluctuation time $\sim 10^{4} \mathrm{~s}$ ) and larger, but not to the $0.001 \mu \mathrm{m}$ grains. The latter are, however, below the detection threshold of the Interstellar Probe instruments and we do not discuss this case in detail. The results for $0.001 \mu \mathrm{m}$ case presented here were obtained using the equilibrium charge.

We restrict our attention to the region of space outside of the heliopause. This makes it possible to use a further approximation of taking the charge of the grain as constant in time. The reason is that the equilibrium charge on the grain does not vary much over this region of space (Fig. 1). We found that this approximation does not significantly affect our results and, by cutting the time needed for calculations, allows us to cover a larger range of parameters.

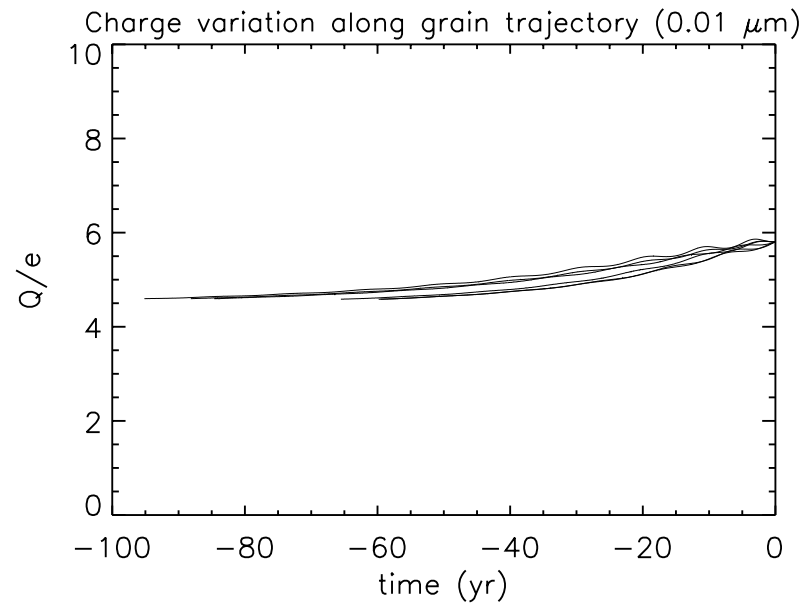

Fig. 1. Grain charge as a function of time along the trajectories of a sample of $0.01 \mu \mathrm{m}$ grains chosen from those used in the calculations of the grain velocity distributions. The trajectories have the same initial point in space (time $t=0$ ) but different initial velocities. The trajectories are calculated in reverse time direction and end when the grains reach the distant region in space within which the phase space distribution function for the grains is assumed known. Note that the charge on the grains (which does not differ from the local value of the equilibrium charge by more than 0.01 percent) does not vary much in the transition region.

In addition to simulations based on full equations of motion, in some calculations (the case of large-scale streaming) we have used the guiding centre approximation (with $Q=$ constant in time). The equations of motion for the guiding centre are (Northrop 1963):

$$
\begin{aligned}
\frac{\mathrm{d} V_{\|}}{\mathrm{d} t}= & -\mu \hat{\boldsymbol{b}} \cdot \nabla \boldsymbol{B}+V_{\|} \boldsymbol{v}_{\mathrm{p} \perp} \cdot(\hat{\boldsymbol{b}} \cdot \nabla) \hat{\boldsymbol{b}} \\
& +\boldsymbol{v}_{\mathrm{p} \perp} \cdot\left(\boldsymbol{v}_{\mathrm{p} \perp} \cdot \nabla\right) \hat{\boldsymbol{b}}+\frac{1}{m} F_{\|} \\
\boldsymbol{V}_{\perp}= & \boldsymbol{v}_{\mathrm{p} \perp}+\boldsymbol{V}_{\perp}^{D} \\
\boldsymbol{V}_{\perp}^{D}= & \frac{m c}{Q B}\left[\frac{v_{\perp}^{\prime 2}}{2} \frac{\boldsymbol{B} \times \nabla B}{B^{2}}+V_{\|}^{2} \hat{\boldsymbol{b}} \times(\hat{\boldsymbol{b}} \cdot \nabla) \hat{\boldsymbol{b}}\right. \\
& +V_{\|} \hat{\boldsymbol{b}} \times\left(\boldsymbol{v}_{\mathrm{p} \perp} \cdot \nabla\right) \hat{\boldsymbol{b}}+V_{\|} \hat{\boldsymbol{b}} \times(\hat{\boldsymbol{b}} \cdot \nabla) \boldsymbol{v}_{\mathrm{p} \perp} \\
& \left.+\hat{\boldsymbol{b}} \times\left(\boldsymbol{v}_{\mathrm{p} \perp} \cdot \nabla\right) \boldsymbol{v}_{\mathrm{p} \perp}-\hat{\boldsymbol{b}} \times \frac{\boldsymbol{F}}{m}\right]
\end{aligned}
$$

with $V_{\|}, V_{\perp}$ the components parallel and perpendicular to $\boldsymbol{B}$ of the velocity of the guiding center, $V_{\perp}$ is the velocity of the drift of the guiding center, $v_{\mathrm{p} \perp}$ the perpendicular plasma velocity, $\boldsymbol{v}_{\perp}^{\prime}=\left(\boldsymbol{v}-\boldsymbol{v}_{\mathrm{p}}\right)_{\perp}$ the grain perpendicular velocity in the plasma frame, $\hat{\boldsymbol{b}}=\boldsymbol{B} / B$ and $\mu=v_{\perp}^{\prime 2} / 2 B$ the adiabatic invariant, which is conserved in this approximation. All quantities are evaluated at the position of the guiding center.

\subsection{Plasma flow and magnetic field}

The plasma velocity $\boldsymbol{v}_{\mathrm{p}}(\boldsymbol{r})$ and the magnetic field $\boldsymbol{B}(\boldsymbol{r})$ used in our simulations were obtained applying a model based on the approach of Nerney et al. (1991, 1993). The plasma flow 
(axially-symmetric relative to the LISM velocity direction and incompressible) is given by an analytic formula (Suess \& Nerney 1990) depending on four parameters: the radius of the solar wind termination shock $R$, the plasma speed at the shock $v_{\mathrm{S}}$, the distance from the Sun to the heliospheric stagnation point $L$, and the LISM plasma speed far upstream $v_{0}=\left|\boldsymbol{V}_{\text {LISM }}\right|$. The values used in our calculations are $R=85 \mathrm{AU}, L=1.5 \mathrm{R}$, $v_{\mathrm{S}}=100 \mathrm{~km} \mathrm{~s}^{-1}$, and $v_{0}=26 \mathrm{~km} \mathrm{~s}^{-1}$. The remaining parameters of the model specify the magnetic field far upstream in the LISM $\boldsymbol{B}_{\text {LISM }}$ and the magnetic field at the termination shock. The magnetic field is assumed to be frozen into the flow.

The model disregards the influence of the magnetic field on plasma flow, which leads to a unrealistically sharp rise of the magnetic field at the heliopause. The "hydrogen wall" (Baranov \& Malama 1993) is not present. In addition, the model has no bow shock, and we have to imitate its effect by an ad hoc procedure (as explained below). The advantage of the model is that the magnetic field can be calculated to any accuracy. Also, the parameters of the model can be changed easily, so that we can explore a range of plausible parameter values. We think that it offers an adequate lowest-order approximation of the forward transition region (the main feature it lacks is the hydrogen wall) and can be used for qualitative discussion of the grain motion in this region. On the other hand, we do not expect that the model gives a good approximation to the global structure of the flow near the heliosphere. The available models based on MHD solutions (Ratkiewicz et al. 1998, 2000; Linde et al. 1998; Pogorelov \& Matsuda 1998; Tanaka \& Washimi 1999) are, however, still incomplete (in particular, the neutral component is not fully included).

We consider two orientations of the magnetic field in the LISM: (1) $\boldsymbol{B} \perp \boldsymbol{V}_{\text {LISM }}$ and (2) magnetic field inclined at $45^{\circ}$ with respect to $\boldsymbol{V}_{\text {LISM. }}$. In each case we take $\left|\boldsymbol{B}_{\text {LISM }}\right|=3.5 \mu \mathrm{G}$. If the bow shock is included in the simulation, we use the following procedure. We assume that the bow shock has the form of a paraboloid surface, and restrict the calculations to the region downstream of it. The plasma flow in this region we take to be given by the analytic formula of Suess \& Schmahl (1990), with the parameter $v_{0}$, representing the LISM velocity, reduced by a factor of 2 to imitate the velocity drop at the bow shock. (That is, the plasma flow velocity used in the simulations for the bow shock case differs from that assumed for the no bow shock case by the parameter $v_{0}$ ). The distance Sun-bow shock is $390 \mathrm{AU}$.

We do not include the effects of turbulence and waves on the grain dynamics, since the relevant parameters in the heliospheric transition region are not known. Our results correspond to the assumption that the relevant mean free paths (in particular, the parallel mean free path for pitch-angle scattering) are, for the grain sizes considered, not less than the characteristic size scale of the transition region.

\subsection{Velocity distributions}

The usual method of deriving the phase space distributions by starting from an initial (usually uniform) distribution of particles and integrating the equations of motion forward in time requires a very large number of trajectories. One problem is that one does not typically know beforehand how to choose the initial region: to calculate the velocity distribution at a given point in space it would be preferable to calculate only the trajectories passing through this point. Calculating in reverse time direction solves this problem.

The method is particularly simple for the case when the Liouville theorem in the phase space holds. The value of the distribution function $F(\boldsymbol{x}, \boldsymbol{v})$ is then constant along the trajectory. To find the value of the distribution function $F$ at $(\boldsymbol{x}, \boldsymbol{v})$ it is then enough to calculate the trajectory passing through $(\boldsymbol{x}, \boldsymbol{v})$ until it reaches the point $\left(\boldsymbol{x}^{\prime}, \boldsymbol{v}^{\prime}\right)$ at which the distribution function is known: the value of $F$ at $(x, v)$ is then given by the known value at $\left(\boldsymbol{x}^{\prime}, \boldsymbol{v}^{\prime}\right)$. Note that moving from the point $(\boldsymbol{x}, \boldsymbol{v})$ (at which the distribution function is to be found) to the point $\left(\boldsymbol{x}^{\prime}, \boldsymbol{v}^{\prime}\right)$ (where the distribution is known) will typically correspond to moving in the reverse time direction. The complete velocity dependence of the distribution function at the point $\boldsymbol{x}$ can be obtained by calculating the trajectories passing through $\boldsymbol{x}$ with different velocities.

The Liouville theorem is valid for the case when the grain charge is constant in time, but it also can be easily shown to apply to the case when the grain charge in Eq. (1) is a given function of the position in space. For the charging rates used in our simulations, the equilibrium charge was space- but not velocity-dependent. This, together with the fact that the grain charges in our simulations were either constant or stayed very close to the equilibrium charge (Fig. 1) allows us to use the Liouville theorem.

To derive the velocity distributions of the grains at a given point in space we therefore use the method of integrating the grains equation of motion backward in time, until the boundary of the integration region is reached. We assume that the distribution function at any point of the boundary is known. It is then used to determine the value of the distribution function at the initial point of the grain trajectory.

The boundary is a surface in the LISM enclosing the forward part of the heliopause at the distance large enough to assume that the velocity distribution of the grains is the same as in the undisturbed LISM. If the bow shock is present, the outer boundary includes the shock surface. We assume that the velocity distribution of the grains is unchanged by crossing the shock. In the present calculations the boundary was assumed to consist of a paraboloidal surface in front of the heliopause $z=-R+(390 \mathrm{AU}-R)\left((w / 700 \mathrm{AU})^{2}-1\right)$ (representing the bow shock surface if the bow shock was present), a cyllindrical surface $w=1400 \mathrm{AU}$ enclosing the sides and the downstream boundary at $z=1000 \mathrm{AU}$. Here the $z$ axis is directed along the LISM velocity direction (with the Sun at $z=0$ ), $w$ is the distance from the $z$ axis and $R=85 \mathrm{AU}$ is the Sun-termination shock distance.

We have assumed that the grain spatial distribution at the boundary (and in the distant LISM) is uniform and that the velocity distributions are narrow in the plasma frame, so that the grains move intially close to the plasma velocity. The form of the velocity distribution we have assumed to be Maxwellian with the width $2-3 \mathrm{~km} \mathrm{~s}^{-1}$. The value of the width was chosen mostly for convenience, since more narrow distributions 
would require calculating more trajectories. Also, to describe the evolution of the initially very narrow distribution the model should include also small-scale effects (like magnetic field inhomogeneities). Finally, we do not expect the error of measurement to be much below $1 \mathrm{~km} \mathrm{~s}^{-1}$. In the present calculations we didn't consider the case of initially wide velocity distributions.

For large grains some of the trajectories cross the heliopause. Our model is not well adapted to describe such trajectories, because its magnetic field at the heliopause is unrealistically high. In addition, our approximation of time-independent equilibrium charge is then incorrect, because the equilibrium charge in the shocked solar wind region is much higher than outside the heliopause. To estimate the resulting errors we have compared the velocity distributions derived using all particle trajectories with those obtained by excluding the trajectories passing through the enhanced field region.

When the charge of the grain is not taken to be constant in time, the method of integrating the equations of motion backwards in time encounters problems. This is because Eq. (2) has a stable fixed point at the equilibrium value of the grain charge, which for the inverse time direction becomes unstable. A partial solution is to reverse the signs of the charging rates. This makes the fixed point unstable (stable for negative time direction) so that, during propagation in the reverse time direction, the grain charge stays near the equilibrium value. We have used this method for some calculations involving 0.001 and $0.01 \mu \mathrm{m}$ grains.

\section{Grains velocity distributions relative to spacecraft in the forward transition region}

The discussed mission concept for an interstellar probe (Mewaldt \& Liewer 2001) suggests a spacecraft travelling in an interstellar upwind direction with radial speed of more than $10 \mathrm{AU}$ per year. The spacecraft would be spin-stabilized with its spin axis tilted relative to the stellar wind direction. Hence a dust detector that measures the mass and the impact speed of the grain would also provide information on the dust velocity vector through the orientation of the spacecraft at the time of the dust impact. Assuming that the number density of interstellar grains with radius larger than $a$ is $\sim a^{-2.5}$ (Mathis et al. 1977) and that the number density of the $a>0.001 \mu \mathrm{m}$ grains is of the order of $10^{-9} \mathrm{~cm}^{-3}$, the flux of the grains of $0.01 \mu \mathrm{m}$ radii relative to spacecraft would be of the order of $2 \times 10^{-5}$ per cm per second.

We estimate the impact distributions of the grains relative to the spacecraft assuming that the observer is moving at $60 \mathrm{~km} \mathrm{~s}^{-1}$ away from the Sun, in the direction near to (offset by a small angle from) that of the Sun velocity relative to LISM. The results presented in this Section correspond to the offset angle $=15^{\circ}$ with the plane defined by the Sun velocity and the spacecraft direction taken to be perpendicular to the $\left(\boldsymbol{B}_{\text {LISM }}, \boldsymbol{V}_{\text {LISM }}\right)$ plane. The observation points are taken to be outside of the heliopause (away from the region where the magnetic field in the model is unrealistically high).

The charges on the grains in most simulations are assumed to be time-independent. Although we label the results with the grain radii $a$ in $\mu \mathrm{m}$, the values of the charge are not (except for $0.001 \mu \mathrm{m}$ and $0.01 \mu \mathrm{m}$ grains) derived by using a detailed model for size-dependent charging rates. Rather, for the grains with $a \geq 0.01 \mu \mathrm{m}$ we use a simple scaling rule: $Q / m \sim a^{-2}$, corresponding to taking a constant potential on the grains, with $a=0.01 \mu \mathrm{m}$ corresponding to $Q=5 \mathrm{e}$ and $m=7.0 \times 10^{6} m_{\mathrm{p}}$ (which follows from the model charging rates for the case of the distant LISM). For $0.001 \mu \mathrm{m}$ grains the simulations use equilibrium charge derived from the model charging rates based on the approach of Kimura \& Mann (1998). We have also used the equilibrium charge in some simulations for the $0.01 \mu \mathrm{m}$ grains and found that the results are not significantly different from those for constant charge.

A conclusion independent of the details of the model and which may be expected to be valid in a general case concerns the effect of the bow shock. The grains would initially move together with the mean LISM flow. On approaching the transition region the plasma flow would be disturbed. The effect on the motion of the small grains depends significantly on the existence of a sharp bow shock.

If there is a bow shock and if its thickness is small (compared to the distance traversed by a grain during one Larmor rotation period), a grain will cross the shock without loss in velocity, while the plasma flow will slow down. The result, assuming that the field is not approximately perpendicular to the bow shock surface, will be an increase in the Larmor rotation radius and in the rigidity of the grain. If the magnetic field is inclined with respect to the shock surface, the grains will also start streaming along the field line relative to plasma motion. If the bow shock does not exist, or if its thickness is large (as suggested by Mullan \& Arge 1996) the plasma velocity change can be regarded as adiabatically slow and there will be no significant difference in transverse velocities generated between the plasma and the small grains. Observation of the streaming or large transverse velocity relative to plasma for small grains just outside the heliopause would then indicate the presence of a sharp bow shock.

Figure 2 shows the impact speed $|v|$ distributions for small grains $(0.001 \mu \mathrm{m}$ to $0.15 \mu \mathrm{m})$ for the case of a sharp bow shock. The velocity distribution in the LISM was taken to be a Maxwellian (in the plasma frame) with the width $3 \mathrm{~km} \mathrm{~s}^{-1}$. The $0.001 \mu \mathrm{m}$ grains are representative of the grains small enough so that $T_{\mathrm{L}} \ll T_{\mathrm{f}}$. The $|v|$ distributions have the same form also for $0.01 \mu \mathrm{m}$ grains $\left(T_{\mathrm{L}}<T_{\mathrm{f}}\right)$ and for $0.015 \mu \mathrm{m}$ grains. Above this limit the distributions become size-dependent, until, for still larger grain size, the distributions take a single-peak form corresponding to the grains moving at the speed close to that of the LISM. The onset of sharp size-dependence of the velocity distributions would indicate that the grain charge/mass ratio crosses the limit corresponding to Larmor rotation time becoming equal to the flow time.

Figure 3 shows the distributions for the case without a sharp bow shock. For all grain sizes considered these have a single narrow peak, but the position of the peak shifts with the grain size. Again there are two regions where the distributions are size-independent: small grains $(0.001 \mu \mathrm{m}$ to $0.02-0.03 \mu \mathrm{m})$, with the peak speed close to the local plasma speed, and larger grains $(0.05-0.15 \mu \mathrm{m})$, for which the peak speed is close to the LISM speed. 


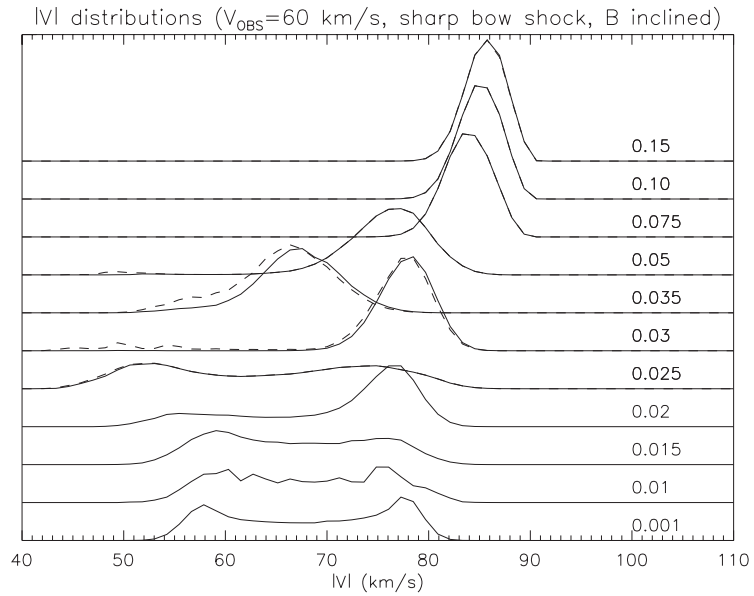

Fig. 2. Simulated distributions of grain impact speed $|v|$ outside the heliopause (distance from the Sun $150 \mathrm{AU}$, observer speed $60 \mathrm{~km} \mathrm{~s}^{-1}$ relative to Sun, direction of motion offset from the apex by $15^{\circ}$ perpendicular to the magnetic field) for the case of sharp bow shock and the IS field inclined at $45^{\circ}$ relative to $V_{\text {LISM }}$. The stacked curves correspond to grain sizes from $0.001 \mu \mathrm{m}$ to $0.15 \mu \mathrm{m}$ as indicated in the figure. The dashed lines show the results if the grain trajectories passing very close to the heliopause are included. The grain velocity distribution in the ISM was assumed to be a Maxwellian (in plasma frame) of width $3 \mathrm{~km} \mathrm{~s}^{-1}$, independent of the grain size. The simulated distributions for small size grains are wide, reflecting Larmor rotation. For $0.001 \mu \mathrm{m}$ grains (for which the Larmor rotation time $T_{\mathrm{L}}$ is much smaller than the flow time $T_{\mathrm{f}}$ through the transition region) and for $0.01 \mu \mathrm{m}$ grains (for which $T_{\mathrm{f}} / T_{\mathrm{L}} \sim 5$ ) the distributions are very similar. This holds also for $0.015 \mu \mathrm{m}$ grains. For $0.02 \mu \mathrm{m}$ grains, the Larmor rotation time is close to the flow time: $T_{\mathrm{f}} / T_{\mathrm{L}} \sim 1$ and the distributions become size-dependent. For large size grains $(0.075$, 0.10 and $0.15 \mu \mathrm{m}$ ) the distributions approach another size-independent form, with single peak at the LISM velocity.

Figures 4-6 present the impact direction distributions as latitude-longitude plots. The directions are represented in terms of angles $\delta$ ("latitude") and $\psi$ ("longitude") defined in the coordinate system $\hat{\boldsymbol{b}}, \hat{\boldsymbol{t}}, \hat{\boldsymbol{v}}$ where $\hat{\boldsymbol{v}}$ is the unit vector along the observer velocity, $\hat{b}$ the projection of the LISM magnetic field direction on the plane perpendicular to $\hat{\boldsymbol{v}}$ and $\hat{\boldsymbol{t}}=\hat{\boldsymbol{b}} \times \hat{\boldsymbol{v}}$ is the "polar" axis. The observer is moving in the $\delta=0^{\circ}, \psi=0^{\circ}$ direction. The projection of the magnetic field is horizontal. One degree of angle corresponds to approximately $1 \mathrm{~km} \mathrm{~s}^{-1}$ transverse velocity (taking the observer speed equal to $60 \mathrm{~km} \mathrm{~s}^{-1}$ ).

Figures 4 and 6 show the distributions respectively for the cases with sharp bow shock and without bow shock. The Larmor ring distribution is seen in Fig. 4. Note also the speed component parallel to the magnetic field (shift from $\psi=0$ ) of about $12 \mathrm{~km} \mathrm{~s}^{-1}$ in the sharp bow shock case and significantly less in the absence of the shock. Figure 5 illustrates the case when the magnetic field in the LISM is perpendicular to the velocity direction. The grains in this case have low parallel velocity component. The Larmor ring distribution is visible from the side.

The behaviour of the velocity distributions for the case of sharp bow shock can be understood as follows. Assuming that the grains move initially close to the velocity of the LISM, the grains emerge from the bow shock as a beam of particles

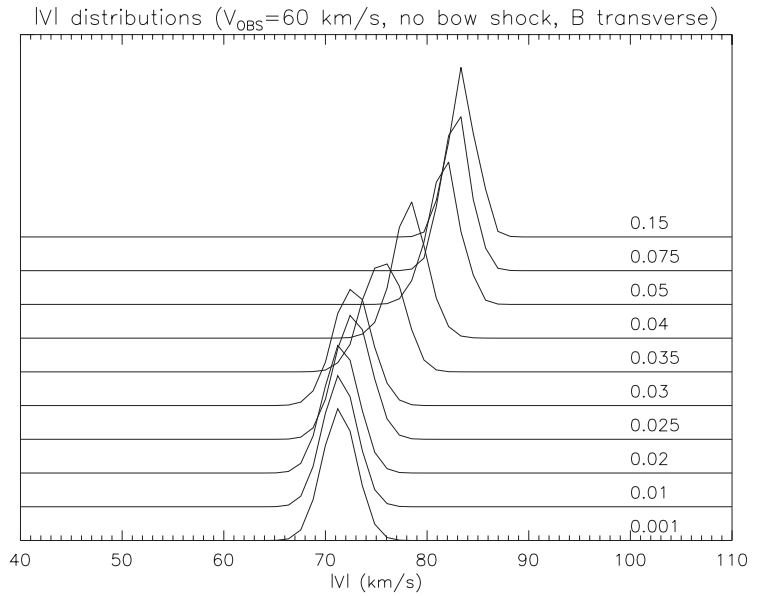

Fig. 3. As previous figure, but for the case of no bow shock and the IS field perpendicular to the LISM velocity. The distributions are narrow and singly peaked. They are independent of the grain size for the cases of small and large grains. There is a transition region where the average impact velocity shifts from the one corresponding to local plasma velocity (small grains) to the LISM velocity (large grains). The distributions for the case of inclined field are similar.

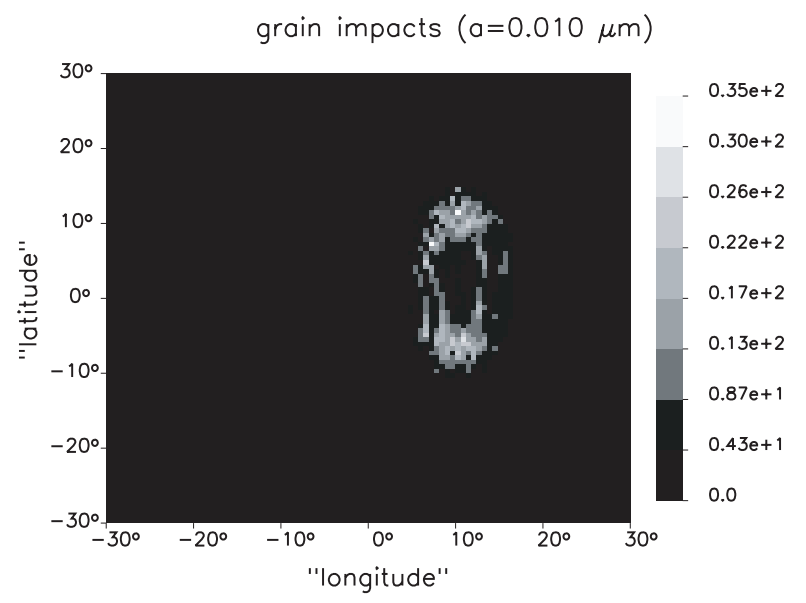

Fig. 4. Simulated distribution over impact directions (the case of sharp bow shock) for small $(a=0.01 \mu \mathrm{m})$ grains as seen by the detector with a field-of-view of $\left( \pm 30^{\circ}\right)^{2}$ situated outside the heliopause at the distance of $150 \mathrm{AU}$ from the Sun, and moving radially in the direction offset from the apex by $15^{\circ}$ perpendicular to the magnetic field, at the speed of $60 \mathrm{~km} \mathrm{~s}^{-1}$. The ring distribution is visible. The magnetic field in the LISM is inclined at $45^{\circ}$ to the LISM velocity. Its projection onto the instrument field-of-view is along the horizontal axis.

moving at the speed of the order of $\sim 10 \mathrm{~km} \mathrm{~s}^{-1}$ relative to plasma. If the Larmor radius of the grains is much smaller than the distance to the point where the velocity distribution of the grains is measured, the observed distribution would have a ring form. If the Larmor radius is much larger, the observed distribution would correspond to the initial beam. For intermediate case, the beam would be deflected, but there would be no fully developed Larmor ring. Apart from Larmor rotation, in the case of inclined field there would be a significant parallel velocity component relative to local plasma flow, because the beam velocity at the shock would then include a parallel component. 


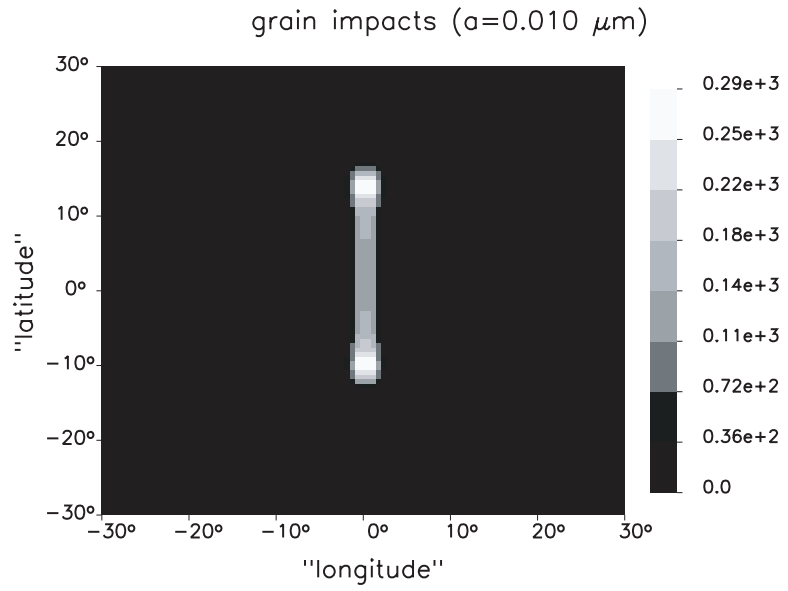

Fig. 5. Simulated impact direction distribution for $0.01 \mu \mathrm{m}$ grains for the case of sharp bow shock, with the magnetic field in the LISM perpendicular to the LISM velocity. In this case the grains do not acquire significant parallel velocity. The Larmor ring is seen side-on.

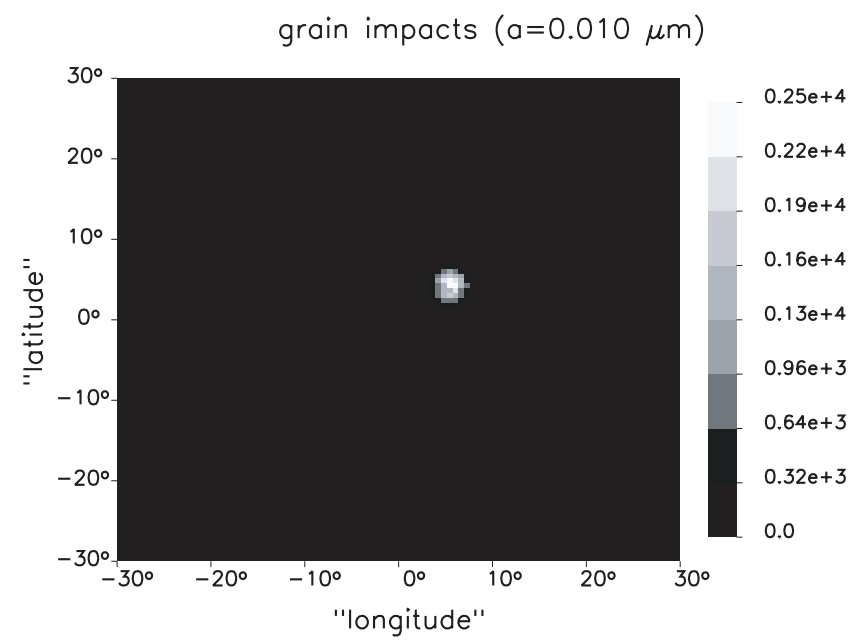

Fig. 6. Simulated impact direction distribution (the case of no bow shock, field inclined at $45^{\circ}$ ) for $0.01 \mu \mathrm{m}$ grains. The grain velocity distribution in the LISM is assumed to be a Maxwellian with the halfwidth of $2 \mathrm{~km} \mathrm{~s}^{-1}$.

For the case with no sharp shocks in the transition region, our simulations suggest that there will be no ring distributions, but that the velocity distributions of the grains would have the form of a narrow beam. For the case of Larmor rotation time much smaller than the time needed for the passage of the grains through the transition region, the grain velocity would stay close to the local plasma velocity. For large Larmor time, the grain velocity would be close to the LISM velocity far upstream. Even for the case of inclined magnetic field, there would be no significant parallel component in the grain velocity distributions in the plasma frame.

\section{Magnetic field and large scale streaming}

Moving through the LISM, the heliosphere forces away the interstellar plasma, keeping it outside the boundary surface. This also affects the motion of small interstellar grains, which are coupled effectively to plasma flow by electromagnetic forces.
If there is a sharp bow shock, the grains crossing the shock acquire a speed difference relative to plasma. The interaction between the grains and the heliosphere is then also modified, with the motion of grains around the heliosphere becoming significantly different from plasma flow.

In our first approach to this problem (Czechowski \& Mann 2001) we have considered the density distribution of the grains in the vicinity of the heliopause and found that the density of small grains is enhanced at the flanks of the heliosphere, in the plane parallel to the magnetic field. Here we look at the same problem using the velocity distributions. We found that the guiding center approximation is a convenient framework in this case. The grain drifts can be seen to be small, so that the motion of the grains can be understood in terms of the guiding centre motion along the magnetic field line, combined with the convection of the magnetic field line by the plasma flow. Note that we neither consider the grains which move very close to the heliopause nor those which cross it: however, for small grains this would be a small minority of the incoming population. In particular, to avoid overestimating the effect, we have included only the field lines for which the magnetic field enhancement does not exceed a factor of $\sim 2$ (the upper limit considered by Holzer 1989; see also Zank 1999),

We calculated the parallel velocity distributions at equally spaced points along a field line passing close (but not too close, to avoid the region of unrealistically large magnetic field) to the heliopause. The calculations were done for a set of field lines corresponding to the subsequent positions in time of a field line transported with the flow. Figures 7, 8 show the results for the field lines already some distance downstream from the stagnation point region, because the streaming of the grains in this case is better developed. The grain velocity distribution in the LISM was taken to be given by the plasma frame Maxwellian with $2 \mathrm{~km} \mathrm{~s}^{-1}$ width.

The results are:

(1) For the perpendicular field case, the grains are accelerated away from the maximum field point of the field line and acquire parallel velocities of the order up to $20 \mathrm{~km} \mathrm{~s}^{-1}$. This results in the grains streaming along a field line away from the heliopause. The streaming grains pass over the slow moving ones, creating the density enhancements (the density wave) on the flanks of the heliosphere.

(2) For the inclined field, the grains start (at the bow shock) with the parallel velocity already of the order of $20 \mathrm{~km} \mathrm{~s}^{-1}$. On approaching the heliopause, they meet with the barrier of high magnetic field, in result of which some are reflected and start moving along the field line in the opposite direction. This leads to a density wave.

These conclusions agree with our calculations of spatial density of grains based on solving numerically the full equations of motion for a large number of particles and analysing the result by statistical method. However, it is possible that more realistic models of the heliosphere would produce a different picture of large-scale streaming. The published results are not detailed enough to clarify the structure of the magnetic field in the transition region, resulting from the MHD simulations. It seems, however, that the field enhancement near to the stagnation point is not large (the magnetic pressure along the 

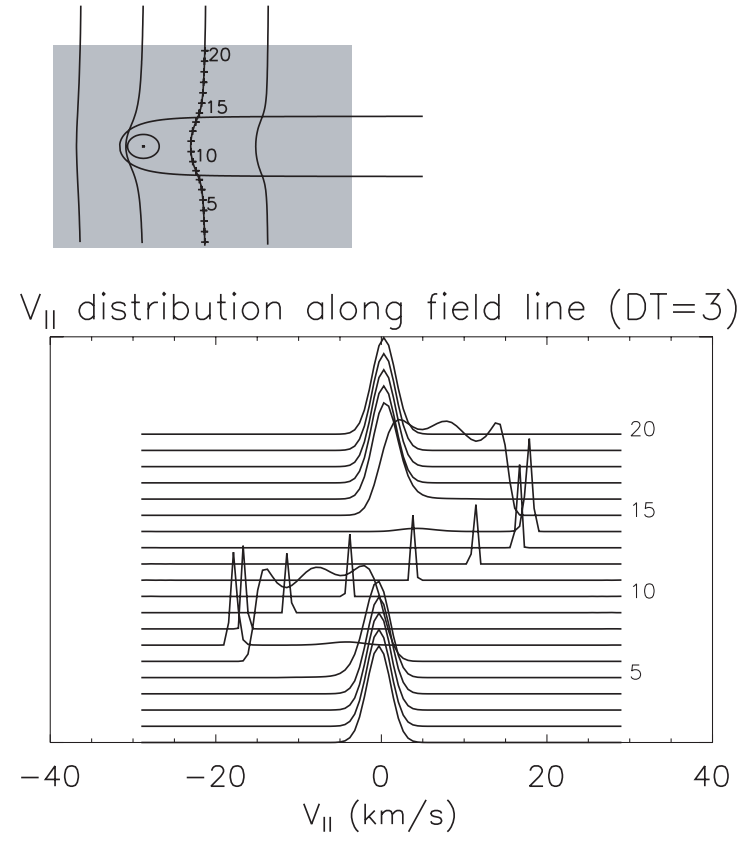

Fig. 7. The $V_{\|}$distributions of the grains along a magnetic field line (the case of the perpendicular field). The distributions shown are for a set of 20 equally spaced points along a field line passing close to the outside surface of the heliopause (see the insert). The maximum field intensity corresponds to the middle of the stack. Note the streams of the grains moving in opposite directions away from the region of high magnetic field, and the density enhancements.

stagnation line increasing by only a factor of 2.5 between the downstream side of the bow shock and the heliopause, Washimi $\&$ Tanaka 1996). From the contour plots in Linde et al. (1998) the magnitude of the field near the heliopause can be seen to reach the values larger than $4.5 \mu \mathrm{G}, 3$ times larger than the assumed LISM value of $1.5 \mu \mathrm{G}$, even away from the stagnation point region. The shape of the field lines is illustrated in Ratkiewicz et al. (2000) suggesting that in the heliotail region the field lines spread away from each other along the heliopause surface, which can lead to lowering of the field magnitude in this region.

Another point is that, since our results were obtained for the large-scale smooth magnetic field, they can be significantly affected by taking into account the interaction with the magnetic field inhomogeneities. In particular, pitch-angle scattering could invalidate the simple streaming picture, if the mean free path is shorter than the characteristic size scale of the heliosphere.

\section{Conclusion}

We have discussed, using numerical simulations, the question of how the passage through the heliospheric transition region would affect the velocity distributions of small interstellar grains, with particular attention to the forward part of the transition region, along the possible trajectory of an Interstellar Probe. Our main conclusion is that measurements of the velocity distributions of small grains, which could be performed during an Interstellar Probe mission, would be a valuable source
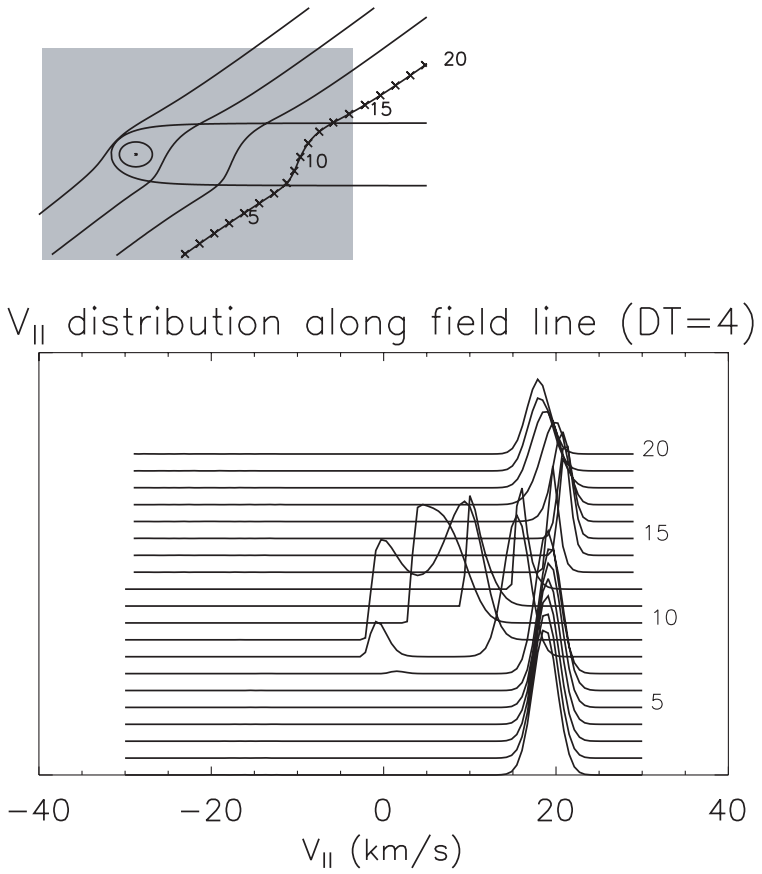

Fig. 8. As previous figure, but for the case of inclined field. Note the reflected streams of the grains reaching $V_{\|}=0$.

of information about the structure of the transition region. The data would in particular indicate the presence of a heliospheric bow shock or of other sharp shock waves. Aside from providing the size distribution of dust in the interstellar medium they would also allow one to derive the charge-to-mass ratio of interstellar grains.

The possibility of deriving conclusions from dust measurements depends critically on the value of the flux intensity of the interstellar grains relative to the spacecraft. We estimate the flux of $0.01 \mu \mathrm{m}$ grains to be of the order of $2 \times 10^{-5} \mathrm{~cm}^{-2} \mathrm{~s}^{-1}$. With the detector size of $(10 \mathrm{~cm}) \times(10 \mathrm{~cm})$ the number of impacts would be of the order of $1.5 \times 10^{2}$ per day. This should make it possible to derive a one-variable distribution function, like the distribution in the impact speed, which may provide information about the existence of the bow shock. The case of the charge-to-mass ratio is more difficult, since it requires comparing the distributions for even smaller grains. If the number of grains above radius $a$ is $\sim a^{-2.5}$ the number of impacts would be of the order of one per two days for the $0.1 \mu \mathrm{m}$ grains. If the efficiency of the detector would be high, a reasonable approximation to the distribution function in impact speed could be obtained after half a year. In this time the spacecraft would move by a distance of $6 \mathrm{AU}$, which is small compared to the size of the transition region. Consequently, the impact speed distribution function can be expected not to vary much over the time needed for the measurement.

We also considered a possible global picture of the motion of grains near the heliopause, following from a simple model. The results suggest that the presence of a sharp bow shock, combined with the draping effect for the magnetic field at the heliopause, can initiate ordered streaming of small $(\sim 0.01 \mu \mathrm{m})$ interstellar grains in the vicinity of the heliosphere, leading also to moderate (a factor of three) grain density enhancement on 
the flanks of the heliopause. This density wave should be distinguished from the variations of the density of small grains caused by the variations in plasma density, in particular at the heliospheric bow shock and in the hydrogen wall region.

Passage through the transition region would also lead to a difference in the velocity distributions between small and bigger grains. The relative velocities between the different dust components would be of the order of $10 \mathrm{~km} \mathrm{~s}^{-1}$ (including also the effect of ordered streaming discussed above). However, due to small dust densities compared to the extension of this dust cloud around the heliopause, mutual dust collisions are negligible.

These qualitative results also apply to astrospheres and we are planning to extend this study to the case of nearby stars.

Acknowledgements. A.C. acknowledges support from the grant KBN 8T12E 029 20. I.M. was supported by the German Aerospace Center DLR (Deutsches Zentrum fuer Luft- und Raumfahrt) under project "Mikro-Impakte: Empirische Analyse der Physik von Hochgeschwindigkeitseinschlaegen" (RD-RX-50 OO0203).

\section{References}

Baranov, V. B., \& Malama, Yu. G. 1993, J. Geophys. Res., 98, 15157 Bertaux, J. L., \& Blamont, J. E. 1976, Nature, 262, 263

Czechowski, A., \& Mann, I. 2001, in The Outer Heliosphere: The Next Frontiers, ed. K. Scherer, H. Fichtner, H. J. Fahr, \& E. Marsch, COSPAR Colloq. Ser. (Pergamon), 365

Fahr, H. J., Kausch, T., \& Scherer, H. 2000, A\&A, 357, 268

Frisch, P. C. 1996, Space Sci. Rev., 78, 213

Frisch, P. C., Dorschner, J. M., Geiss, J., et al. 1999, ApJ, 525, 492

Grün, E., Gustafson, B., Mann, I., et al. 1994, A\&A, 286, 915

Grün, E., Krüger, H., \& Landgraf, M. 2000, in Minor Bodies in the Outer Solar System, ed. A. Fitzsimmons, D. Jewitt, \& R. M. West (New York, Berlin: Springer), 99

Gurnett, D. A., Kurth, W. S., Allendorf, S. C., \& Poynter, R.L. 1993, Science, 262, 199

Holzer, T. E. 1989, ARA\&A, 27, 199

Kimura, H., \& Mann, I. 1998, ApJ, 499, 454

Krüger, H., Grün, E., Landgraf, M., et al. 2001, Planet. Space Sci., 49, 1303

Kurth, W. S., \& Gurnett, D. A. 2003, J. Geophys. Res., in press
Lallement, R., Ferlet, R., Lagrange, A. M., et al. 1995, A\&A, 304, 461 Lallement, R. 2001, Ap\&SS, 277, 205

Levy, E. H., \& Jokipii, J. R. 1976, Nature, 264, 423

Linde, T. J., Gombosi, T. I., Roe, P. L., et al. 1998, J. Geophys. Res., 103,1889

Linde, T. J., \& Gombosi, T. I. 2000, J. Geophys. Res., 105, 10411

Linsky, J. L., \& Wood, B. E. 1996, ApJ, 463, 254

Mann, I. 1996, Space Sci. Rev., 78, 259

Mann, I., \& Kimura, H. 2000, J. Geophys. Res., 105, 10317

Mathis, J. S., Rumpl, W., \& Nordsieck, K. H. 1977, ApJ, 217, 425

Mewaldt, R. M., \& Liewer, P. C., 2001, in The Outer Heliosphere: The Next Frontiers, ed. K. Scherer, H. Fichtner, H. J. Fahr, \& E. Marsch, COSPAR Colloq. Ser. (Pergamon), 451

Mullan, D. J., \& Arge, C. N. 1996, J. Geophys. Res., 101, 2535

Nerney, S., Suess, S. T., \& Schmahl, E. J. 1991, A\&A, 250, 556

Nerney, S., Suess, S. T., \& Schmahl, E. J. 1993, J. Geophys. Res., 98, 15169

Nerney, S., Suess, S. T., \& Schmahl, E. J. 1995, J. Geophys. Res., 100, 3463

Northrop, T. G. 1963, Adiabatic motion of charged particles (New York: Interscience)

Parker, E. N. 1963, Interplanetary Dynamical Processes (New York: Interscience)

Pauls, H. L., Zank, G. P., \& Williams, L. L. 1995, J. Geophys. Res., 100,21595

Pogorelov, N. V. 1995, A\&A, 297, 835

Pogorelov, N. V., \& Matsuda, T. 1998, J. Geophys. Res., 103, 237

Ratkiewicz, R., Barnes, A., Molvik, G. A., et al. 1998, A\&A, 335, 363

Ratkiewicz, R., Barnes, A., \& Spreiter, J. R. 2000, J. Geophys. Res., 105,25021

Scherer, K., \& Fahr, H. J. 2003, Geophys. Res. Lett., 30, 1045

Suess, S. T., \& Nerney, S. 1990, J. Geophys. Res., 95, 6403

Tanaka, T., \& Washimi, H. 1999, J. Geophys. Res., 104, 12605

von Steiger, R., Lallement, R., \& Lee, M. A. (eds.) 1996, The Heliosphere in the Local Interstellar Medium, (London: Kluwer)

Washimi, H., \& Tanaka, T. 1996, Space Sci. Rev., 78, 85

Wenzel, K.-P., Marsden, R. G., Page, D. E., \& Smith, E. J. 1992 , A\&AS, 92, 207

Witte, M., Rosenbauer, H., Banaszkiewicz, M., \& Fahr, H. 1993, Adv. Space Res. 13, 121

Wood, B. E., \& Linsky, J. L. 1998, ApJ, 492, 788

Zank, G. P., Pauls, H. L., Williams, L. L., \& Hall, D. T. 1996, J. Geophys. Res., 101, 21639

Zank, G. P. 1999, Space Sci. Rev., 89, 413 